\title{
Characterization of high healthcare utilizer groups using administrative data from an electronic medical record database
}

Sheryl Hui-Xian Ng${ }^{1}$, Nabilah Rahman ${ }^{1}$, Ian Yi Han Ang², Srinath Sridharan', Sravan Ramachandran, Debby D. Wang', Chuen Seng Tan ${ }^{3}$, Sue-Anne $\operatorname{Toh}^{2}$ and Xin Quan Tan ${ }^{2,3^{*}}$

\begin{abstract}
Background: High utilizers (HUs) are a small group of patients who impose a disproportionately high burden on the healthcare system due to their elevated resource use. Identification of persistent HUs is pertinent as interventions have not been effective due to regression to the mean in majority of patients. This study will use cost and utilization metrics to segment a hospital-based patient population into HU groups.

Methods: The index visit for each adult patient to an Academic Medical Centre in Singapore during 2006 to 2012 was identified. Cost, length of stay (LOS) and number of specialist outpatient clinic (SOC) visits within 1 year following the index visit were extracted and aggregated. Patients were HUs if they exceeded the 90th percentile of any metric, and Non-HU otherwise. Seven different HU groups and a Non-HU group were constructed. The groups were described in terms of cost and utilization patterns, socio-demographic information, multi-morbidity scores and medical history. Logistic regression compared the groups' persistence as a $\mathrm{HU}$ in any group into the subsequent year, adjusting for socio-demographic information and diagnosis history.

Results: A total of 388,162 patients above the age of 21 were included in the study. Cost-LOS-SOC HUs had the highest multi-morbidity and persistence into the second year. Common conditions among Cost-LOS and Cost-LOSSOC HUs were cardiovascular disease, acute cerebrovascular disease and pneumonia, while most LOS and LOS-SOC HUs were diagnosed with at least one mental health condition. Regression analyses revealed that HUs across all groups were more likely to persist compared to Non-HUs, with stronger relationships seen in groups with high SOC utilization. Similar trends remained after further adjustment.

Conclusion: HUs of healthcare services are a diverse group and can be further segmented into different subgroups based on cost and utilization patterns. Segmentation by these metrics revealed differences in socio-demographic characteristics, disease profile and persistence. Most HUs did not persist in their high utilization, and high SOC users should be prioritized for further longitudinal analyses. Segmentation will enable policy makers to better identify the diverse needs of patients, detect gaps in current care and focus their efforts in delivering care relevant and tailored to each segment.
\end{abstract}

Keywords: Healthcare, Utilization, Segmentation, Super-utilizers, Cost, Expenditure, Administrative, Persistence

\footnotetext{
* Correspondence: kyletanxq@gmail.com

${ }^{2}$ Regional Health System Office, National University Health System, Singapore,

Singapore

${ }^{3}$ Saw Swee Hock School of Public Health, National University of Singapore

and National University Health System, Singapore, Singapore

Full list of author information is available at the end of the article
}

(c) The Author(s). 2019 Open Access This article is distributed under the terms of the Creative Commons Attribution 4.0 International License (http://creativecommons.org/licenses/by/4.0/), which permits unrestricted use, distribution, and

reproduction in any medium, provided you give appropriate credit to the original author(s) and the source, provide a link to the Creative Commons license, and indicate if changes were made. The Creative Commons Public Domain Dedication waiver (http://creativecommons.org/publicdomain/zero/1.0/) applies to the data made available in this article, unless otherwise stated. 


\section{Background}

High healthcare utilizers are a small group of patients who impose a disproportionately high burden on the healthcare system due to their elevated resource use, and often have unmet care needs or receive unnecessary care [1]. To design policies to address these issues, high healthcare utilization and its drivers have been studied extensively in recent years. The definition of high utilization has been heterogeneous. The choice of metric used to measure utilization often differs and depends on the disease or health service context. As distributions of healthcare cost and utilization incurred by patients are often skewed [2-4], the approach of defining high utilizers (HUs) as patients in the top percentiles of healthcare cost has been commonly adopted. Most studies use cost to identify HUs as it can be regarded as a measure of utilization intensity $[5,6]$. It also gives a direct economic perspective (e.g. potential cost savings and impact on government funding) to the problem at hand [7]. The percentile threshold for cost used to identify HUs varies between studies, ranging from the top 5\% of patients [7-16] to the top 20\% [17], with top $10 \%$ being the most common definition used $[1,7,11,15,18-26]$. Cost is a good measure of utilization and can serve as a proxy for utilization across different resource types (e.g. inpatient admissions, outpatient visits and procedures). However, as cost would be heavily influenced by the number of inpatient bed days incurred by a patient, looking at cost alone may not provide a complete picture of utilization volume. Other metrics commonly used to identify HUs include outpatient visits to clinics [27], emergency attendances [28-30], and inpatient utilization such as readmissions within a certain period [31-33] or length of stay (LOS) [34-36]. There are few papers that examine multiple metrics simultaneously [23, 37-39]. Examining other metrics of utilization in tandem with cost will allow policymakers and clinicians to look at multiple dimensions of resource use [37, 40], and understand the different underlying drivers to get a more comprehensive understanding of healthcare utilization. Furthermore, segmentation of a patient population using multiple metrics would create smaller groups of patients with largely similar utilization patterns and characteristics, facilitating targeting and tailoring of interventions for effective use of resources [41].

With the increasing adoption of electronic medical record (EMR) systems in hospitals [42-44], comprehensive administrative cost and utilization data over multiple years are now more readily available. Researchers and health systems can use this information to segment the general patient population and address the diverse needs of each patient segment [41]. Segmentation will help identify homogenous patient subpopulations and provide knowledge on their characteristics, needs and trajectories over time. This knowledge would then support development and implementation of interventions targeted at each subpopulation, such that the interventions are more tailored to individual needs, and likely to be of greater impact [41, 45-48]. In the long run, this would also facilitate program evaluation and outcomes tracking for each group [48].

While examining high utilization in a cross-sectional manner allows us to understand the profiles of HUs, observing HUs longitudinally would provide valuable information on how utilization per patient accumulates over time, how patients transit between HU groups and how patients' utilizations change with their transitions. The definition of persistence of HU behavior differs widely between studies, with one definition being recurrence as a $\mathrm{HU}$ in the subsequent year $[4,9,19,49]$. Identification of persistent users is pertinent as interventions on HUs have not been shown to be efficacious or cost-effective potentially due to regression to the mean in majority of patients $[20,50]$. Hence, insights from these longitudinal analyses could potentially inform how healthcare systems can better design and target interventions for HUs.

This study will demonstrate the use of cost and utilization metrics to segment a patient population into groups of high healthcare utilizers, based on 1 year's patterns of hospital-based resource use in an Academic Medical Center (AMC) in Singapore. The groups' sociodemographic characteristics, utilization patterns and medical history will be described for comparison. The comparisons will illustrate the benefits of using multiple metrics to identify different HU profiles, highlight the healthcare needs associated with each profile and their subsequent longitudinal behaviors. This work provides multifaceted insights on the characteristics of high healthcare utilizers, which will inform program and policy development, and the identification of the correct subgroups for more targeted interventions.

\section{Methods}

Data analyzed was from a hospital administrative database in an AMC in Singapore for the period of 2006 to 2013. Ethics approval was obtained from the review board of the healthcare cluster. Details of preparation and processing of the database are described elsewhere [51]. Patients aged 21 years and above and had at least one record of either an inpatient admission, specialist consultation, therapy visit or emergency attendance between 1st January 2006 and 31st December 2012 were included in the study. The index visit to the AMC for each patient, defined as the first visit occurring on or after 1st January 2006 and before 1st January 2013, was identified. All visits within 1 year following the index visit were then extracted. Multi-morbidity measures were included using the Charlson Comorbidity Index 
(CCMI) score adapted by Quan [52, 53], as well as a polypharmacy score (PPS) measuring the highest number of unique dispensed medications a patient was ever prescribed in a visit [54]. Diagnosis codes were aggregated into Clinical Classification Software (CCS) groups for ease of reporting [55].

Metrics of hospital utilization described in this study were the number of inpatient admissions, LOS in inpatient care, Specialist Outpatient Clinic (SOC) visits, Emergency Department (ED) attendances, as well as healthcare costs accumulated over all hospital settings incurred per patient. Healthcare costs incurred by the patient were proxied by the bill charged to the patient before any Government subsidies or third-party payments (e.g. payments by insurers or employers) and were presented in Singapore dollars (S\$), adjusted to 2015 figures. LOS per inpatient admission was computed as the total duration of hospitalization that was not attributed to short stays (e.g. day surgery and endoscopy). This was in line with methodology adopted by the Organization for Economic Co-operation and Development (OECD) where short-term treatments were excluded due to variation in clinical environments for these treatments across countries [56]. SOC visits were defined as outpatient specialist consultations within the AMC. Primary care information was not available at time of the study. For analysis, cost and utilization from all visits within the observed year were aggregated and reported for each patient.

\section{Classification into $\mathrm{HU}$ groups}

With no universal set of metrics to measure high resource use, Cost, LOS and number of SOC visits were selected as the metrics for identification of HUs to capture multiple facets of hospital utilization, and the threshold for high utilization was set at 90th percentile of each metric as it is the most common threshold used in literature. Cost would provide a measure of the global economic burden on the hospital system, including all inpatient-related resources, all ambulatory services including emergency department visits and outpatient visits, manpower, consumables, pharmaceuticals and procedures. To measure multiple aspects of utilization in addition to cost, LOS and SOC visits incurred would also be included, as they measure resource and service use specifically in the inpatient and outpatient settings respectively. Furthermore, we chose to use LOS as a metric of inpatient utilization instead of the number of admissions as LOS would account for the variation in number of patient days per admission, which would more accurately reflect the intensity of inpatient resource use in comparison to using only number of admissions as a metric [57]. We chose not to include number of ED attendances as a specific metric for identification of HUs. As frequent ED users, commonly defined as patients who incur 4 or 5 visits in literature, constitute only $5 \%$ of a patient cohort, the 90 th percentile cut off would capture a substantially larger subgroup and not distinguish the high users sufficiently well [5864]. Only patients with admissions were included in computation of the 90th percentile of LOS. Patients who exceeded the threshold for any of the three metrics were classified as HUs for the observed year. Patients who were HUs in all three metrics were labelled as CostLOS-SOC HUs, while patients who satisfied the criteria in only two metrics were Cost-LOS, Cost-SOC or LOSSOC HUs correspondingly. Patients who satisfied the HU criteria in only a single metric were classified as Cost, LOS or SOC HUs accordingly. This ensured membership in each HU group was mutually exclusive. Patients who incurred cost or utilization below the 90th percentile for all metrics were classified as the Non-HU group.

The patient cohort, partitioned by their HU group memberships, were described in terms of their cost and utilization patterns, and profiled using their sociodemographic information, multi-morbidity scores and medical history. Age at patients' first visit in the system was reported. As patients seeking care in public healthcare institutions in Singapore may receive government subsidies with the quantum dependent on their household income [65], we classified patients into three levels of subsidy based on the amount of subsidised treatment they received. Patients would have received either only subsidised treatment, only unsubsidised treatment, or a mix of both subsidised and unsubsidised treatment. Socio-economic status (SES) of patients was described by their housing type, derived from their last known postal code information in the system. Housing in Singapore can be tiered into private housing, public housing and public rental housing. Private housing caters mainly to the upper-middle to upper income groups. Public housing caters to the middle-class population and public rental housing to the low-income groups. Each residential property or block in Singapore is uniquely assigned a postal code akin to an address, and neither public housing nor public rental housing share postal codes with private housing types. Approximately $80 \%$ of residents in Singapore live in public housing, with the smallest housing type being 1-room rental flats and the largest being executive flats with three bedrooms [66]. As the cost of these flats increase with size, housing type can serve as a proxy of SES. In this study, we identified the housing type present at each postal code using a map of postal codes and their respective housing types validated from a separate study, and for public housing blocks with more than one flat type, the housing type assigned was the flat type with the largest 
proportion in the block [51]. We categorized housing type in increasing order of SES: 1/2-room flats, 3-room flats and larger, and private housing. Hence, given a patient's postal code, we were able to determine their corresponding housing type as a proxy of SES. Multimorbidity measures, cost and utilization were reported in terms of median and interquartile range (IQR) specified as a range spanning the first quartile to the third quartile. The proportion of patients who died within the observed year was also reported. To describe the medical history of each group, the primary diagnosis for each visit (i.e. medical condition the patient sought healthcare service for) was extracted for each patient. We looked at common diagnoses within each diagnosis groups, in terms of both the number of patients who had ever sought care for that condition, as well as separately in terms of the number of visits attributed to that condition. Within each HU group, the five most common conditions recorded were reported.

\section{Persistence of HU behavior}

After describing the demographic and clinical profiles of the HU groups, we were interested in whether the $\mathrm{HU}$ groups had differing extents of persistence in the subsequent year. Persistence was defined as membership in any $\mathrm{HU}$ group in a subsequent observed year. All cost and utilization incurred during a one-year period following the first observed year was aggregated for each patient, and patients were classified as HUs if they exceeded the same HU thresholds from the first observed year. The proportion of persistent patients in each HU group were reported. To compare the tendencies for persistence between the HU groups, logistic regression models were built. Patients who died within the first observed year or had missing socio-demographic information were excluded from the analysis, and patients with no utilization in the subsequent year were subsumed under the Non-HU status. Missing CCMI information was assumed to be 0 . First, a model (Model 0) was constructed to look at the associations between each first observed year HU group and the outcome of subsequent year HU status. Model 1 was estimated by adjusting Model 0 for the socio-demographic characteristics and multi-morbidity measures and removing factors which were not statistically significant at $0.1 \%$ significance level $(p<0.001)$ to obtain a parsimonious model. Model 2 was then built by further adjusting Model 1 for common HU conditions, and similarly factors which were no longer significant at $0.1 \%$ significance level $(p<$ 0.001 ) were removed. Odds ratios (ORs) for each factor and the corresponding 99\% confidence intervals (CIs) were reported. McFadden's pseudo- $\mathrm{R}^{2}$ for each model was reported [67], and likelihood ratio tests (LRTs) were conducted for comparison of model fit between Model 0 and 1, as well as Model 1 and 2 [68]. All statistical analyses were carried out using RStudio using the dplyr package [69, 70].

\section{Results}

A total of 388,162 patients above the age of 21 recorded at least one visit to the hospital over the study period. The patient population was divided into eight distinct segments, with seven groups of HUs and one group of Non-HUs. The utilization patterns of patients according to the HU grouping are described in Table 1. Non-HUs constitute $83 \%$ of all patients and $25 \%$ of all costs during the first observed year. Few patients had inpatient utilization, and outpatient utilization was an average of 1 SOC visit or ED attendance. Cost HUs accounted for almost $16 \%$ of total costs despite constituting less than $4 \%$ of the cohort. The median costs for these HUs was $S \$ 16,591$ and median inpatient utilization was 1 inpatient admission and 7 days of LOS. Similarly, most LOS HUs only had 1 inpatient admission, but their median bill was lower at S\$9073 and LOS was longer at 19 days. LOS-SOC HUs incurred similar bill sizes and inpatient utilization as LOS HUs, but had additional high SOC usage (LOS HU: median: 1 visit; LOS-SOC HU: 9 visits). SOC HUs, due to the large group size (6.7\%), accounted for $8 \%$ of all cost despite having zero inpatient utilization and ED attendances on average. CostSOC HUs generally incurred more utilization in comparison to SOC HUs (median inpatient admissions: 1; LOS: 6 days; SOC visits: 12). Cost-LOS HUs incurred more cost and inpatient utilization than Cost HUs, at a median cost of $\mathrm{S} \$ 31,762$, and median inpatient utilization of 2 admissions and 27 days of LOS. The Cost-LOS-SOC patients incurred the highest utilization across all metrics (median cost: $\$ \$ 49,248$; inpatient admissions: 3; LOS: 29; SOC visits: 13; ED attendances: 2).

The socio-demographic profiles of the HU groups are presented in Table 2. Overall, most Non-HU patients were aged below 40 and had low multi-morbidity (median age: 36; median CCMI: 0; median PPS: 2). The majority were male, Chinese, sought at least some subsidised services or stayed in 3-room public housing or larger (male: 59.6\%; Chinese: 58.0\%; only unsubsidised treatment: 11.7\%; 3-room and larger: 62.6\%). Death within the observed year and persistence was low (death: 1.4\%; persistence: $2.2 \%$ ). Cost HUs in comparison were older, had a larger proportion of patients who sought only unsubsidised treatment and a tenth of the patients died during the year (median age: 55 ; only unsubsidised treatment: 19.7\%). LOS HUs were also older than NonHUs, mostly female, and a larger proportion were Chinese (median age: 55; male: 39.2\%; Chinese: $72.8 \%$ ). Almost all sought at least some subsidised services (99.5\%), and $9.2 \%$ of patients died during the year. LOS- 


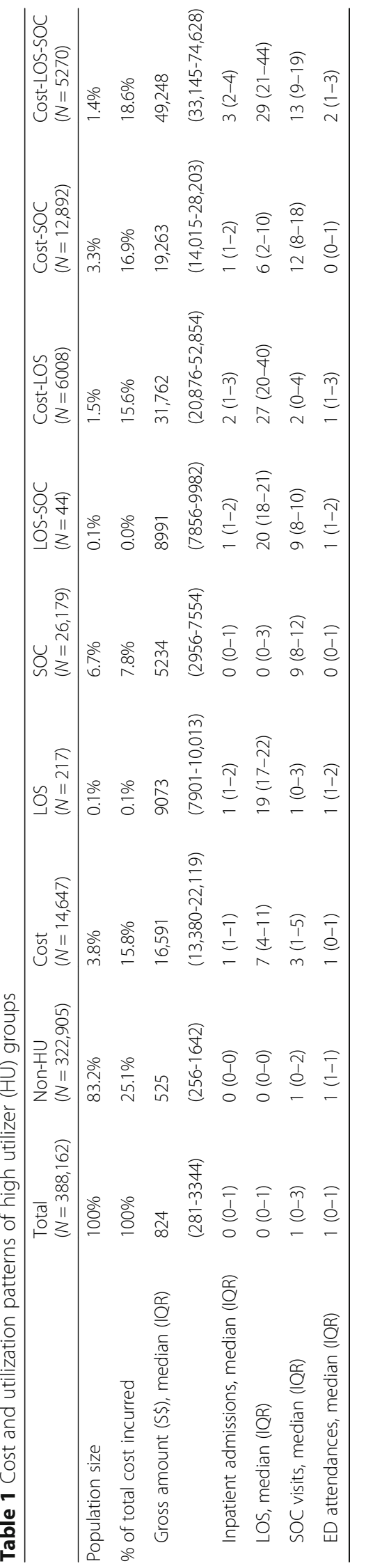




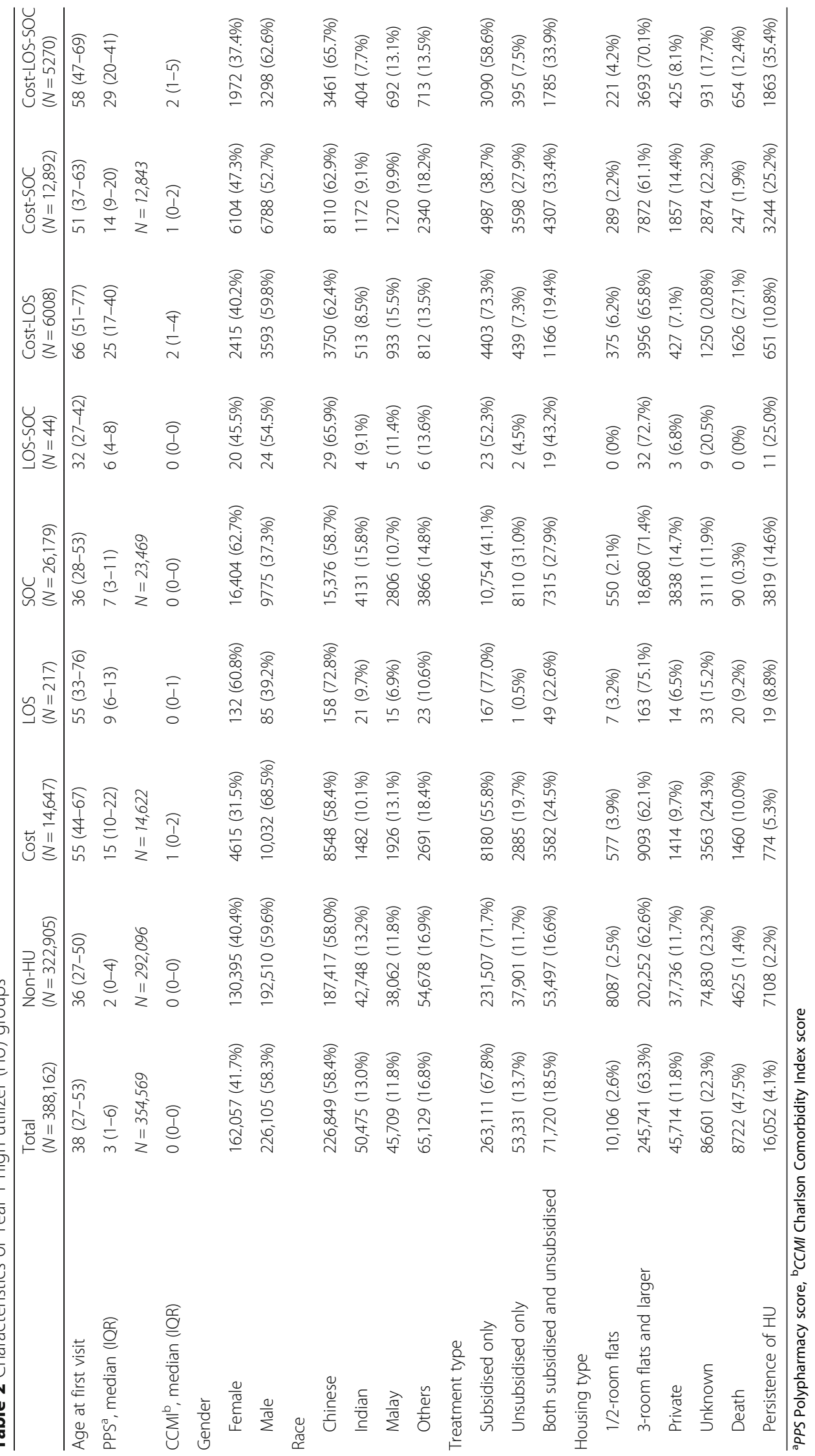


SOC HUs were similar to LOS HUs in terms of race, ethnicity and SES, but were younger with a median age of 32, and all patients survived. SOC HUs were similar in demographic profile to Non-HUs, but had more female patients and the highest proportion of patients who sought only unsubsidised treatment (male: 37.3\%; unsubsidised treatment: $31.0 \%$ ). Persistence was also prevalent in $15 \%$ in SOC HUs, which was substantially higher than in the Non-HUs. Cost-SOC patients were older than SOC HUs, had more males and exhibited more persistence in comparison (median age: 51; male: 52.7\%; persistence: $25.2 \%$ ). Cost-LOS patients were the oldest, had the highest multi-morbidity and the highest proportion of patients living in 1/2-room flats (median age: 66; median CCMI: 2; median PPS: 25; $1 /$ 2-room flat: $6.2 \%)$. They also had the highest death rate among all groups within the first year (27.1\%). Cost-LOS-SOC HUs had the highest multi-morbidity, and a third of patients persisted as HUs into the second year (median CCMI: 2; median PPS: 29; persistence: $35.4 \%)$.

Table 3 illustrates the five most common conditions patients in each $\mathrm{HU}$ group that were ever diagnosed in the first year. External injuries were common primary diagnoses in the Non-HU group. Cardiovascular disease was prevalent among the Cost HUs (Coronary atherosclerosis: 20.1\%; Acute myocardial infarction: 19.6\%). SOC HUs were commonly diagnosed with routine ambulatory conditions, with predominantly pregnancy related conditions (Normal pregnancy: 13.3\%; complications: 4.4\%), while Cost-SOC HUs were commonly diagnosed with complex ambulatory conditions such as cancer and female infertility (Cancer of breast: 7.4\%; Female infertility: 5.0\%). Most LOS and LOS-SOC HUs were diagnosed with at least one mental health condition, with mood disorders highly prevalent (LOS: 24.0\%; LOS-SOC: $52.3 \%$ ). For Cost-LOS and Cost-LOS-SOC HUs, common conditions were cardiovascular disease, acute cerebrovascular disease as well as pneumonia (Cardiovascular: Cost-LOS: $8.2 \%$, CostLOS-SOC: $10.9 \%$; Cerebrovascular: Cost-LOS: $17.5 \%$; Cost-LOS-SOC: 8.3\%; Pneumonia: Cost-LOS: 13.9\%; Cost-LOS-SOC: $7.9 \%)$. Common diagnoses ranked by visit frequency revealed similar trends. The only exception was the prevalent conditions in Cost-LOS-SOC HUs were lymphoma, leukemia, colon cancer and renal failure (Additional file 1).

We then sought to identify factors associated with persisting as a HU into the subsequent year. Generally, persistent and non-persistent HUs differed in sociodemographic characteristics and prevalence of common HU conditions (Additional file 2). Of all patients, 16,052 (4.2\%) patients were $\mathrm{HU}$ in a subsequent year. From Table 4, Model 0 revealed that HUs across all groups were more likely to persist as a $\mathrm{HU}$ in any group in the subsequent year, compared to Non-HUs. The weakest association was seen in the Cost HUs, while the strongest association was seen in the Cost-LOS-SOC HUs (Cost: OR $=2.73$, 99\% CI: 2.45-3.03; Cost-LOS-SOC: 31.59, 99\% CI: 29.02-34.37), with generally stronger associations seen in groups with high SOC utilization (LOS-SOC: 16.72, 99\% CI: 6.26-38.93; Cost-SOC: 16.30, 99\% CI: 15.31-17.35). After adjusting Model 0 for all socio-demographic factors and multi-morbidity scores, housing type was removed from the model due to lack of statistical significance. The resulting model, Model 1, revealed that the trends in persistence among the $\mathrm{HU}$ groups had remained but decreased in strength across all groups except for LOS-SOC HUs (Model 0: OR: 16.72, 99\% CI: 6.26-38.93; Model 1: 17.28, 99\% CI: 6.39-40.94). The LRT revealed that Model 1 exhibited significantly better fit than Model $0(p<0.001)$. Adjusting Model 1 for the 26 common HU conditions and further refining the model to retain only statistically significant factors, only 13 conditions remained in Model 2. The same trend in varying tendencies in persistence among the HU groups was observed. A diagnosis of breast cancer, mood disorders, hypertension or female infertility was also associated with a higher likelihood of persistence (Cancer of breast: 1.28, 99\% CI: 1.09-1.51; mood disorders: 1.45, 99\% CI: 1.11-1.85; hypertension: $1.53,99 \%$ CI: 1.36-1.71; female infertility: $1.72,99 \%$ CI: $1.39-2.12$ ). The LRT revealed that Model 2 exhibited significantly better fit than Model $1(p<0.001)$.

\section{Discussion}

HUs of healthcare services are a diverse group and can be further segmented into different subgroups based on utilization metrics available in most hospital administrative databases, such as cumulative cost, length of stay or outpatient visits. Segmentation by these utilization metrics revealed differences in socio-demographic characteristics, varying persistence in high utilization and distinct variations in disease profiles of patients. Our results showed that the HU groups exhibit differences in age and comorbidity. High-cost groups were generally older and of higher multi-morbidity in comparison to the lowcost groups, which is consistent with other studies associating older age and multi-morbidity with higher healthcare costs $[1,3,71-73]$. As few studies define high utilization using multiple metrics simultaneously $[37,40]$, our study adds meaningful insights into the characteristics of patients in different HU groups, such as the variation in extent of multi-morbidity between the groups. However, while socio-demographic factors have been shown to be associated with HUs in other populations $[9,14,17,74]$, this was not as 
Table 3 Top 5 common conditions in Year 1 high utilizer (HU) groups

\begin{tabular}{|c|c|c|}
\hline HU Group & Condition & Patients ever diagnosed (\%, \\
\hline \multirow[t]{5}{*}{ Non-HU } & Superficial injury; contusion & $27,049(8.4 \%)$ \\
\hline & Other upper respiratory infections & $14,778(4.6 \%)$ \\
\hline & Sprains and strains & $14,546(4.5 \%)$ \\
\hline & Non-infectious gastroenteritis & $12,371(3.8 \%)$ \\
\hline & Open wounds of extremities & $11,447(3.5 \%)$ \\
\hline \multirow[t]{5}{*}{ Cost } & Coronary atherosclerosis and other heart disease & $2946(20.1 \%)$ \\
\hline & Acute myocardial infarction & $2877(19.6 \%)$ \\
\hline & Acute cerebrovascular disease & $879(6.0 \%)$ \\
\hline & Essential hypertension & $781(5.3 \%)$ \\
\hline & Fracture of lower limb & $604(4.1 \%)$ \\
\hline \multirow[t]{5}{*}{ LOS } & Mood disorders & $52(24.0 \%)$ \\
\hline & Schizophrenia and other psychotic disorders & $36(16.6 \%)$ \\
\hline & Residual codes; unclassified & $17(7.8 \%)$ \\
\hline & Pneumonia $^{a}$ & $13(6.0 \%)$ \\
\hline & Intracranial injury & $12(5.5 \%)$ \\
\hline \multirow[t]{5}{*}{ SOC } & Normal pregnancy and delivery & $3490(13.3 \%)$ \\
\hline & Cataract & $1379(5.3 \%)$ \\
\hline & Other complications of birth & $1151(4.4 \%)$ \\
\hline & Fracture of upper limb & $1055(4.0 \%)$ \\
\hline & Gastritis and duodenitis & $743(2.8 \%)$ \\
\hline \multirow[t]{5}{*}{ LOS-SOC } & Mood disorders & $23(52.3 \%)$ \\
\hline & Schizophrenia and other psychotic disorders & $14(31.8 \%)$ \\
\hline & Other eye disorders & $5(11.4 \%)$ \\
\hline & Superficial injury; contusion & $5(11.4 \%)$ \\
\hline & Burns & $4(9.1 \%)$ \\
\hline \multirow[t]{5}{*}{ Cost-LOS } & Acute cerebrovascular disease & $1049(17.5 \%)$ \\
\hline & Pneumonia $^{\mathrm{a}}$ & $838(13.9 \%)$ \\
\hline & Urinary tract infections & $546(9.1 \%)$ \\
\hline & Coronary atherosclerosis and other heart disease & $493(8.2 \%)$ \\
\hline & Acute myocardial infarction & $417(6.9 \%)$ \\
\hline \multirow[t]{5}{*}{ Cost-SOC } & Cancer of breast & $955(7.4 \%)$ \\
\hline & Coronary atherosclerosis and other heart disease & $925(7.2 \%)$ \\
\hline & Female infertility & $650(5.0 \%)$ \\
\hline & Fracture of upper limb & $619(4.8 \%)$ \\
\hline & Essential hypertension & $581(4.5 \%)$ \\
\hline \multirow[t]{5}{*}{ Cost-LOS-SOC } & Coronary atherosclerosis and other heart disease & $572(10.9 \%)$ \\
\hline & Acute cerebrovascular disease & $440(8.3 \%)$ \\
\hline & Pneumonia $^{a}$ & $414(7.9 \%)$ \\
\hline & Essential hypertension & $398(7.6 \%)$ \\
\hline & Septicemia (except in labour) & $363(6.9 \%)$ \\
\hline
\end{tabular}

aexcept that caused by tuberculosis or sexually transmitted disease

apparent in our population as housing type, as a proxy of SES, does not appear to be a differentiating factor across the groups.
Our results also revealed that the HU groups have different disease profiles. The disease profile of the CostLOS and Cost-LOS-SOC HUs, together with the older 
Table 4 Factors associated with persistence as a HU into a subsequent year

\begin{tabular}{|c|c|c|c|}
\hline & Odds ratio $(99 \%$ cor & & \\
\hline & Model 0 & Model $1^{c}$ & Model $2^{c}$ \\
\hline Non-HU & 1.00 & 1.00 & 1.00 \\
\hline Cost & $2.73(2.45-3.03)$ & $1.85(1.64-2.07)$ & $1.77(1.57-1.98)$ \\
\hline LOS & $5.67(2.92-9.97)$ & $3.60(1.83-6.44)$ & $3.36(1.71-6.02)$ \\
\hline SOC & $8.20(7.75-8.68)$ & $6.71(6.31-7.12)$ & $7.40(6.95-7.87)$ \\
\hline LOS-SOC & $16.72(6.26-38.93)$ & $17.28(6.39-40.94)$ & $14.04(5.07-34.39)$ \\
\hline Cost-LOS & $7.41(6.55-8.35)$ & $4.07(3.51-4.71)$ & $3.97(3.42-4.61)$ \\
\hline Cost-SOC & $16.30(15.31-17.35)$ & $9.83(9.08-10.63)$ & $9.43(8.68-10.25)$ \\
\hline Cost-LOS-SOC & $31.59(29.02-34.37)$ & $17.61(15.50-20.01)$ & $16.82(14.78-19.15)$ \\
\hline Age at first visit & & $1.01(1.01-1.02)$ & $1.01(1.01-1.01)$ \\
\hline $\mathrm{CCMI}^{\mathrm{b}}$ & & $1.27(1.25-1.29)$ & $1.24(1.22-1.26)$ \\
\hline $\mathrm{PPS}^{\mathrm{a}}$ & & $0.99(0.99-0.99)$ & $0.99(0.99-1.00)$ \\
\hline Gender & & & \\
\hline Female & & 1.00 & 1.00 \\
\hline Male & & $0.71(0.68-0.75)$ & $0.72(0.69-0.75)$ \\
\hline Race & & & \\
\hline Chinese & & 1.00 & 1.00 \\
\hline Indian & & $1.23(1.14-1.33)$ & $1.26(1.17-1.36)$ \\
\hline Malay & & $1.02(0.95-1.09)$ & $1.05(0.97-1.12)$ \\
\hline Others & & $0.93(0.86-1.01)$ & $0.93(0.86-1.00)$ \\
\hline Nationality & & & \\
\hline Foreigner & & 1.00 & 1.00 \\
\hline Singaporean & & $2.35(2.21-2.51)$ & $2.27(2.12-2.42)$ \\
\hline Treatment type & & & \\
\hline Subsidised only & & 1.00 & 1.00 \\
\hline Both subsidised and unsubsidised & & $1.42(1.34-1.51)$ & $1.46(1.37-1.55)$ \\
\hline Unsubsidised only & & $1.89(1.78-2.01)$ & $1.85(1.74-1.97)$ \\
\hline Common HU conditions & & & \\
\hline Other puerperium complications of birth & & & $0.35(0.26-0.47)$ \\
\hline Normal pregnancy and delivery & & & $0.41(0.34-0.49)$ \\
\hline Open wounds of extremities & & & $0.48(0.38-0.59)$ \\
\hline Fracture of upper limb & & & $0.48(0.39-0.58)$ \\
\hline Burns & & & $0.51(0.28-0.84)$ \\
\hline Fracture of lower limb & & & $0.66(0.54-0.80)$ \\
\hline Other eye disorders & & & $0.70(0.56-0.87)$ \\
\hline Cataract & & & $0.70(0.59-0.82)$ \\
\hline Superficial injury contusion & & & $0.70(0.61-0.79)$ \\
\hline Cancer of breast & & & $1.28(1.09-1.51)$ \\
\hline Mood disorders & & & $1.45(1.11-1.85)$ \\
\hline Essential hypertension & & & $1.53(1.36-1.71)$ \\
\hline Female infertility & & & $1.72(1.39-2.12)$ \\
\hline Model comparison indices & & & \\
\hline McFadden's $\mathrm{R}^{2}$ & 0.16 & 0.20 & 0.21 \\
\hline Residual deviance & 111,630 & 106,351 & 105,539 \\
\hline
\end{tabular}


Table 4 Factors associated with persistence as a HU into a subsequent year (Continued)

\begin{tabular}{llll}
\hline & \multicolumn{2}{l}{ Odds ratio (99\% confidence interval) } & ${\text { Model } 2^{c}}^{c}$ \\
\cline { 2 - 4 } & Model 0 & Model 1 & 379,404 \\
\hline Residual degrees of freedom & 379,427 & 379,417 & $<0.001$ \\
Likelihood ratio test $\left(x^{2}\right)$ & - & $<0.001$ & $<$ \\
\hline
\end{tabular}

${ }^{\mathrm{a} P P S}$ Polypharmacy score, ${ }^{\mathrm{b}} \mathrm{CCMI}$ Charlson Comorbidity Index score, ${ }^{\mathrm{C}}$ Model 1: Adjusted for socio-demographics and multi-morbidity; Model 2: Adjusted for sociodemographics, multi-morbidity and common $\mathrm{HU}$ conditions

average age, higher CCMI and substantial death rate, suggest a frail elderly archetype similar to clusters with advanced age and high prevalence of complex chronic conditions found in recent segmentation studies [39, 75 , 76]. The finding of acute cardiac events as a one-off high-cost condition was consistent with other studies. Similarly, common resource-intensive conditions such as cancers and renal failure were observed to be among the most prevalent conditions in the Cost-SOC and CostLOS-SOC HUs when the number of visits per condition was taken into account $[13,19,26]$. The LOS and LOSSOC HUs were found to be primarily patients with diagnosed mental illness. The underlying drivers of high utilization and required interventions for patients admitted to the psychiatric wards and patients admitted to the general wards would differ. For patients admitted for psychiatric conditions, interventions such as rapid psychiatric review upon admission could potentially reduce inpatient stay in the psychiatric wards [77, 78]. For the patients admitted for non-psychiatric diagnoses, the long stay may be driven by factors such as poor access to appropriate psychosocial care [37], suggesting that instead of cost reduction measures, patients may instead benefit from an integrated model of care to reduce the burden on acute inpatient care $[79,80]$.

A key finding is that most HUs in our patient cohort do not persist in their high utilization, precluding intervention after identification in the first observed year where they incur substantial resource use. This finding is consistent with other studies that have shown that even within HUs, there exists a small group of high-risk persistent users incurring a disproportionate amount of cost $[7,20,73,81,82]$. HUs were more likely to recur as a $\mathrm{HU}$ in any group in the subsequent year, and this phenomenon was more pronounced in groups with high SOC utilization in the first observed year. Our multivariable model suggested that patients incurring high resource usage in the SOC setting, such as for treatment of female infertility, should be prioritized for further longitudinal analyses to better understand their utilization trajectories, with the aim of developing programs with these specific characteristics in mind. In addition, current disease management processes for hypertension and mood disorders should also be flagged for further analyses and refinement to address the tendency for persistence in these patients. Future studies would also seek to examine persistence of $\mathrm{HU}$ behavior over a longer duration and the different trajectories of each subgroup to inform intervention design and targeting.

As utilization patterns may be driven by patients' disease type, progression and management [73], interventions to reduce excess resource use are currently disease-specific and in context of the usual disease management process [83-85]. However, we found that certain conditions are prevalent across multiple HU groups, suggesting that the traditional disease-centred programs may be capturing a group of patients with the same diagnosis but with heterogeneity in utilization patterns and by extension, care needs. Such disease-centric programs may then be limited in effectiveness due to the inherent variation present in the patient populations treated and the hospital setting in which the disease is treated. For instance, diagnosis of acute cerebrovascular disease was found to be prevalent across the Cost, CostLOS and Cost-LOS-SOC HU groups. Care pathways have been commonly adopted for stroke management to improve patient care quality and outcomes [86]. While these programs may include outpatient treatment as part of the pathway, they generally focus on inpatient-related care during the acute phase. However, it is clear that there is a group of patients with cerebrovascular disease that have high outpatient needs, suggesting the need to look at a more holistic program that focuses not only on the inpatient aspect of stroke care, but extends to outpatient care as well for this group.

An effective program design should either accommodate the variation in the patient profiles, or target only a particular subgroup of patients. As patients at risk of high utilization often have high prevalence of multiple complex chronic conditions and not just one disease, new integrated models of care that are generic and disease agnostic, and that address the cross-cutting needs of a patient, may be more appropriate and effective in addressing high resource use across the different archetypes of HUs. Interventions such as case management, care planning and bundling of care have already been implemented in specific high-risk groups with complex needs such as older patients and patients with chronic diseases [87-92]. However, with increasing age, chronicity and complexity in the general population, applying this patient-centric approach across the different segments of the population will be better able to address 
the diverse health and social needs of each group [92, 93]. In parallel, the empirical approach in segmenting the patient population we have proposed would facilitate targeting of certain subgroups, by increasing withingroup homogeneity in utilization profile and subsequently the relevance of any new interventions targeted at reducing high resource use.

Our findings highlight the importance of selecting the correct metrics in population segmentation. Selection can either be hypothesis-driven, with the intention to zoom in on a particular type of patient group, or pragmatically motivated by availability and access to information. Segmentation of patient populations is commonly achieved using clustering, but the segments have to be labelled post hoc given the characteristics of the identified clusters [39, 75, 76, 94, 95]. On the other hand, Cost, LOS and SOC data are convenient starting points for segmentation since they constitute the basic data collected for hospital databases and can be readily processed to generate intuitive and reproducible $\mathrm{HU}$ groups based on the 90th percentile of the cohort. While cost is a straightforward metric of resource use, broadening the definitions to include other metrics and further stratifying these HUs unveils the elevated resource use in other areas that would have been obscured. This representation of other non-high-cost HU groups highlight potential areas for improvement in current care processes which would have otherwise been missed, should the focus only be on high-cost groups.

Originating from one of the only two AMCs in Singapore, the data and analysis offers an important overview of HUs in an AMC in an Asian population. All patients above the age of 21 were included for analysis and information was collected at point of visit, minimising selection and information bias. Socio-demographic information was only available for the last visit in the system, and as changes to gender and ethnicity of the patients over the study period would have been minimal, only non-differential misclassification biases due to changes in housing type would be an inherent limitation in interpreting the information on SES of the patients. The reported healthcare costs in this study were estimated using patient bills as a proxy of cost, and do not reflect the true costs incurred by the hospital. However, as patient bills have been demonstrated to be positively correlated to costs across various studies [96-98], these billed charges are nonetheless a valid measure for the purpose of identifying high utilizers in our study. The use of observation years instead of calendar years allowed us to better account for resource use arising from disease progression over time. The associations seen between the first observed year HU groups and persistence of $\mathrm{HU}$ would only be generalizable to patients who survived the year. This study provides an extensive but incomplete comparison and description of HUs, as primary care data was not included, and we were not able to examine the implications of segmentation on primary care utilization. As the healthcare system in Singapore was reorganized in 2017, a group of polyclinics was integrated into the healthcare cluster and the inclusion of this primary care data for future work would complete the picture of $\mathrm{HU}$ groups in the cluster. Utilization of patients in other healthcare clusters was also not available, which would underestimate the total healthcare utilization accumulated by patients who seek care across multiple hospitals. A local study on three regional hospitals found that the rate of patients visiting all three hospitals was $8 \%$, suggesting the need to take into account potential cross-utilization of patients in interpretation of our findings [99]. Generalizability of the characteristics of HUs to non-tertiary care settings would also be limited given that the study was based on an AMC. Taking into consideration the abovementioned limitations, this study nonetheless adds invaluable insight into the use of administrative data to segment a hospital-based patient population, and the profiles of patients with varying utilization patterns across the different hospital settings.

An extension of the segmentation approach illustrated in this study would be to segment a specific clinical subpopulation, examine the HU group distribution in this subpopulation, and compare these distributions across different clinical diagnoses. Further studies would also seek to expand on the persistence of HUs into subsequent years and distinguish the trajectories for each $\mathrm{HU}$ group. Effective identification and targeting of persistent users would maximise the use of resources channelled to these interventions, as patients who will revert to low resource use on their own over time will be omitted, and only patients who remain within the system and require the intervention will receive the program. These persistent users could be characterised and distinguished from the transient HUs, with the aim of informing program design to detect and target persistence in context of each group's utilization patterns and disease profile. In addition, as we have examined the $\mathrm{HU}$ behaviour from the health system perspective in this study, a follow-up study examining patients with high out-of-pocket expenditure would be conducted to provide insight on high utilization from the patient's perspective.

\section{Conclusion}

High utilizers are a heterogeneous group of patients and there is a need to move beyond a one-size-fits-all metric to measure high utilization. We demonstrated the use of healthcare cost, as well as LOS and SOC utilization as metrics to identify different $\mathrm{HU}$ groups in a cohort of patients followed for 1 year. Differences in socio- 
demographic characteristics, multi-morbidity and disease profile were detected between the HU groups. Persistence of HU behavior in our study was pronounced in groups with high SOC utilization, and this trend was evident even after accounting for socio-demographic and clinical characteristics. These groups with high SOC utilization would be prime candidates for in-depth analysis of longitudinal behavior to distinguish persistent HUs from transient HUs, track their transitions to different HU groups in subsequent years, and determine groups feasible for intervention. Intervention design tackling excess resource use should take into consideration the inherent variation in utilization patterns among the patients and address the specific needs of each subgroup when developing an effective and targeted program. Segmentation of a patient cohort using these utilization metrics will enable policy makers to better identify the diverse needs of patients, detect gaps in current care and focus their efforts in delivering care relevant and tailored to each segment.

\section{Additional files}

Additional file 1: Top 5 common conditions in Year 1 high utilizer (HU) groups by visit frequency. (DOCX $19 \mathrm{~kb}$ )

Additional file 2: Descriptive statistics for persistent and non-persistent high utilizers (HUs). (DOCX 19 kb)

\section{Abbreviations \\ AMC: Academic Medical Center; CCMI: Charlson Comorbidity Index; CCS: Clinical Classification Software; Cl: Confidence interval; ED: Emergency Department; EMR: Electronic medical record; HU: High utilizer; IQR: Interquartile range; LOS: Length of stay; LRT: Likelihood ratio test; OECD: Organisation for Economic Co-operation and Development; OR: Odds ratio; PPS: Polypharmacy score; SES: Socio-economic status; SOC: Specialist Outpatient Clinic}

\section{Acknowledgements}

Not applicable.

\begin{abstract}
Authors' contributions
XQT and SHXN conceptualised the study. SS, SR, DDW, NR and SHXN processed the database for analysis. SHXN performed the segmentation and subsequent analyses, and drafted the manuscript. NR, IYHA and XQT were major contributors in writing the manuscript. SAT and TCS provided revisions and approval for the manuscript. All authors read and approved the final manuscript.
\end{abstract}

\section{Funding}

The work is co-funded by the National University Health System and National University of Singapore. The grant CF/SCL/16/025 was awarded to XQT for 'Health Innovation Programme - Tackling The Challenge of High-cost Healthcare Users'. The funders had no role in study design, data collection and analysis, decision to publish, or preparation of the manuscript.

\section{Availability of data and materials}

The datasets generated and/or analysed during the current study are not publicly available due to the Personal Data Protection Act enacted in Singapore, which prohibits the disclosure of identifiable data to the public. $\mathrm{R}$ programming codes used in the analysis are available from the corresponding author on reasonable request.

\section{Ethics approval and consent to participate}

The database used in this study was approved as a National Healthcare Group (NHG) Domain Specific Review Board (DSRB) Standing Database (NUSSSHSPH/2015-00032). Ethics approval and waiver of consent for this study was obtained from DSRB (Reference Number: 2016/01011).

\section{Consent for publication}

Not applicable.

\section{Competing interests}

The authors declare that they have no competing interests.

\section{Author details}

${ }^{1}$ Centre for Health Services and Policy Research, Saw Swee Hock School of Public Health, National University of Singapore and National University Health System, Singapore, Singapore. ${ }^{2}$ Regional Health System Office, National University Health System, Singapore, Singapore. ${ }^{3}$ Saw Swee Hock School of Public Health, National University of Singapore and National University Health System, Singapore, Singapore.

Received: 27 December 2018 Accepted: 10 June 2019

Published online: 05 July 2019

\section{References}

1. JJG W, Van Der WPJ, MAC T, Westert GP, PPT J. Systematic review of highcost patients characteristics and healthcare utilisation. BMJ Open. 2018;8. https://doi.org/10.1136/bmjopen-2018-023113.

2. Reid R, Evans R, Barer M, Sheps S, Kerluke K, McGrail K, et al. Conspicuous consumption: characterizing high users of physician services in one Canadian province. J Health Serv Res Policy. 2003;8:215-24. https://doi.org/ 10.1258/135581903322403281.

3. Calver J, Bramweld KJ, Preen DB, Alexia SJ, Boldy DP, KA MC. High-cost users of hospital beds in Western Australia: A population-based record linkage study. Med J Aust. 2006;184:393-7.

4. Moturu ST, Johnson WG, Liu H. Predictive risk modelling for forecasting high-cost patients: a real-world application using Medicaid data. Int J Biomed Eng Technol. 2010;3(1/2):114. https://doi.org/10.1504/JJBET.2010. 029654.

5. Diehr P, Yanez D, Ash A, Hornbrook M, Lin DY. Methods for analyzing health care utilization and costs. Annu Rev Public Health. 1999;20:125-44.

6. Heslop L, Athan D, Gardner B, Diers D, Poh BC. An analysis of high-cost users at an Australian public health service organization. Heal Serv Manag Res. 2005;18:232-43.

7. Coughlin TA, Long SK. Health care spending and service use among highcost Medicaid beneficiaries, 2002-2004. Inquiry. 2009:46:405-17.

8. Chechulin Y, Nazerian A, Rais S, Malikov K. Predicting patients with high risk of becoming high-cost healthcare users in Ontario (Canada). Healthc Policy. 2014;9:68-79.

9. Fitzpatrick T, Rosella LC, Calzavara A, Petch J, Pinto AD, Manson H, et al. Looking beyond income and education: Socioeconomic status gradients among future high-cost users of health care. Am J Prev Med. 2015;49:16171. https://doi.org/10.1016/j.amepre.2015.02.018.

10. Guilcher SJTT, Bronskill SE, Guan J, Wodchis WP. Who are the high-cost users? A method for person-centred attribution of health care spending. PLoS One. 2016;11:1-15. https://doi.org/10.1371/journal.pone.0149179.

11. Hayes SL, Salzberg C, McCarthy D, Radley DC, Abrams MK, Shah T, et al. High-need, High-cost patients: Who are they and how do they use health care? 2016.

12. Hunter G, Yoon J, Blonigen DM, Asch SM, Zulman DM. Health care utilization patterns among high-cost VA patients with mental health conditions. Psychiatr Serv. 2015;66:952-8. https://doi.org/10.1176/appi.ps. 201400286.

13. Pritchard D, Petrilla A, Hallinan S, Taylor DH, Schabert VF, Dubois RW. What contributes most to high health care costs? Health care spending in high resource patients. J Manag Care Spec Pharm. 2016;22:102-9. https://doi.org/ 10.18553/jmcp.2016.22.2.102.

14. Rosella LC, Fitzpatrick T, Wodchis WP, Calzavara A, Manson H, Goel V. Highcost health care users in Ontario, Canada: demographic, socio-economic, and health status characteristics. BMC Health Serv Res. 2014;14:532. https:// doi.org/10.1186/s12913-014-0532-2. 
15. Wodchis WP, Austin PC, Henry DA. A 3-year study of high-cost users of health care. Can Med Assoc J. 2016;188:182-8.

16. Zulman DM, Chee CP, Wagner TH, Yoon J, Cohen DM, Holmes TH, et al. Multimorbidity and healthcare utilisation among high-cost patients in the US veterans affairs health care system. BMJ Open. 2015;5:1-10.

17. Lemstra M, Mackenbach J, Neudorf C, Nannapaneni U. High health care utilization and costs associated with lower socio-economic status: results from a linked dataset. Can J Public Heal Can Sante'e Publique. 2009;100:180-3.

18. Lu J, Britton E, Ferrance J, Rice E, Kuzel A, Dow A. Identifying future high host individuals within an intermediate cost population. Qual Prim Care. 2015;23:318-26

19. Joynt KE, Gawande AA, Orav EJ, Jha AK. Contribution of preventable acute care spending to total spending for high-cost. J Am Med Assoc. 2013;309: 2572-8.

20. Yoon J, Chee CP, Su P, Almenoff P, Zulman DM, Wagner TH. Persistence of high health care costs among VA patients. Health Serv Res. 2018:1-19. https://doi.org/10.1111/1475-6773.12989.

21. Boult C, Kessler J, Urdangarin C, Boult L, Yedidia P. Identifying workers at risk for high health care expenditures: A short questionnaire. Dis Manag. 2004;7: 124-35

22. Liptak GS, Shone LP, Auinger P, Dick AW, Ryan SA, Szilagyi PG. Short-term persistence of high health care costs in a nationally representative sample of children. Pediatrics. 2006;118:e1001-9. https://doi.org/10.1542/peds.20052264.

23. Reichard A, Gulley SP, Rasch EK, Chan L. Diagnosis isn't enough: Understanding the connections between high health care utilization, chronic conditions and disabilities among U.S. working age adults. Disabil Health J. 2015;8:535-46. https://doi.org/10.1016/j.dhjo.2015.04.006.

24. Robst J. Comparing methods for identifying future high-cost mental health cases in medicaid. Value Heal. 2012;15:198-203. https://doi.org/10.1016/j.jval. 2011.08 .007

25. Sen B, Blackburn J, Aswani MS, Morrisey MA, Becker DJ, Kilgore ML, et al. Health expenditure concentration and characteristics of high-cost enrollees in CHIP. Inquiry. 2016;53:1-9.

26. Beaulieu ND, Joynt KE, Wild R, Jha AK. Concentration of high-cost patients in hospitals and markets. Am J Manag Care. 2017;23:233-238.

27. Lin JD, Loh CH, Choi IC, Yen CF, Hsu SW, Wu JL, et al. High outpatient visits among people with intellectual disabilities caring in a disability institution in Taipei: A 4-year survey. Res Dev Disabil. 2007;28:84-93.

28. Blank FSJ, Li H, Henneman PL, Smithline HA, Santoro JS, Provost D, et al. A descriptive study of heavy emergency department users at an academic emergency department reveals heavy ED users have better access to care than average users. J Emerg Nurs. 2005;31:139-44.

29. Capp R, Kelley L, Ellis P, Carmona J, Lofton A, Cobbs-Lomax D, et al. Reasons for frequent emergency department use by medicaid enrollees: a qualitative study. Acad Emerg Med. 2016;23:476-81.

30. Billings J, Raven MC. Dispelling an urban legend: Frequent emergency department users have substantial burden of disease. Health Aff. 2013;32: 2099-108.

31. Howell S, Coory M, Martin J, Duckett S. Using routine inpatient data to identify patients at risk of hospital readmission. BMC Health Serv Res. 2009;9:1-9.

32. Petrey LB, Weddle RJ, Richardson B, Gilder R, Reynolds M, Bennett M, et al. Trauma patient readmissions: Why do they come back for more? J Trauma Acute Care Surg. 2015;79:717-25.

33. Fabbian F, Boccafogli A, De Giorgi A, Pala M, Salmi R, Melandri R, et al. The crucial factor of hospital readmissions: A retrospective cohort study of patients evaluated in the emergency department and admitted to the department of medicine of a general hospital in Italy. Eur J Med Res. 2015; 20:1-6.

34. Cyganska M. The impact factors on the hospital high length of stay outliers. Procedia Econ Financ. 2016;39:251-5. https://doi.org/10.1016/S22125671(16)30320-3

35. Freitas A, Silva-Costa T, Lopes F, Garcia-Lema I, Teixeira-Pinto A, Brazdil P, et al. Factors influencing hospital high length of stay outliers. BMC Health Serv Res. 2012;12:265

36. Marcin JP, Slonim AD, Pollack MM, Ruttimann UE. Long-stay patients in the pediatric intensive care unit. Crit Care Med. 2001;29:652-7. http://www.ncbi. nlm.nih.gov/pubmed/11373438.

37. Wick JP, Hemmelgarn BR, Manns BJ, Tonelli M, Quan H, Lewanczuk R, et al. Comparison of methods to define high use of inpatient services using population-based data. J Hosp Med. 2017;12:596-602. https://doi.org/10. 12788/jhm.2778.

38. Lee NS, Whitman N, Vakharia N, Taksler GB, Rothberg MB. High-cost patients: Hot-spotters don't explain the half of it. J Gen Intern Med. 2017;32:28-34.

39. Vuik SI, Mayer E, Darzi A. A quantitative evidence base for population health: Applying utilization-based cluster analysis to segment a patient population. Popul Health Metr. 2016;14:1-9. https://doi.org/10.1186/s12963-016-0115-z.

40. Nguyen OK, Tang N, Hillman JM, Gonzales R. What's cost got to do with it? Association between hospital costs and frequency of admissions among "high users" of hospital care. J Hosp Med. 2013;8:665-71.

41. Vuik SI, Mayer EK, Darzi A. Patient segmentation analysis offers significant benefits for integrated care and support. Health Aff. 2016;35:769-75.

42. Murdoch TB, Detsky AS. The inevitable application of big data to health care. JAMA. 2013;309:1351-2. https://doi.org/10.1001/jama.2013.393.

43. Dean BB, Natoli JL, Nordyke RJ. Use of electronic medical records for health outcomes research. Med Care Res Rev. 2009;66:611-38.

44. Lin J, Jiao T, Biskupiak JE, McAdam-Marx C. Application of electronic medical record data for health outcomes research: a review of recent literature. Expert Rev Pharmacoecon Outcomes Res. 2013;13:191-200. https://doi.org/ 10.1586/erp.13.7.

45. Jensen PB, Jensen $L$, Brunak S. Mining electronic health records: Towards better research applications and clinical care. Nat Rev Genet. 2012;13:395405. https://doi.org/10.1038/nrg3208.

46. Hillestad R, Bigelow J, Bower A, Girosi F, Meili R, Scoville R, et al. Can electronic medical record systems transform health care? Potential health benefits, savings, and costs. Health Aff. 2005;24:1103-17.

47. Vuik SI, Mayer E, Darzi A. Enhancing risk stratification for use in integrated care: A cluster analysis of high-risk patients in a retrospective cohort study. BMJ Open. 2016;6:1-8.

48. Chong JL, Matchar DB. Benefits of population segmentation analysis for developing health policy to promote patient-centred care. Ann Acad Med Singapore. 2017;46:287-9.

49. Monheit AC. Persistence in health expenditures in the short run: Prevalence and consequences. Med Care. 2003;41:|l|153-II|64.

50. Chakravarty S, Cantor JC. Informing the design and evaluation of superuser care management initiatives. Med Care. 2016;54:860-7. https://doi.org/10. 1097/MLR.0000000000000568.

51. Rahman N, Wang DD, Hui-Xian Ng S, Ramachandran S, Sridharan S, Khoo A, et al. Processing of electronic medical records for health services research in academic medical centre: methods and validation. JMIR Med Informatics. 2018. https://doi.org/10.2196/10933.

52. Charlson ME, Pompei P, Ales KL, MacKenzie CR. A new method of classifying prognostic comorbidity in longitudinal studies: development and validation. J Chronic Dis. 1987;40:373-83.

53. Quan H, Sundararajan V, Halfon P, Fong A, Burnand B, Luthi JC, et al. Coding algorithms for defining comorbidities in ICD-9-CM and ICD-10 administrative data. Med Care. 2005:43:1130-9.

54. Masnoon N, Shakib S, Kalisch-Ellett L, Caughey GE. What is polypharmacy? A systematic review of definitions. BMC Geriatr. 2017;17:1-10.

55. Agency for Healthcare Research and Quality. Clinical Classifications Software (CCS) for ICD-9-CM. 2017. www.hcup-us.ahrq.gov/toolssoftware/ccs/ccs.jsp.

56. Drösler SE, Romano PS, Tancredi DJ, Klazinga NS. International comparability of patient safety indicators in 15 OECD member countries: A methodological approach of adjustment by secondary diagnoses. Health Serv Res. 2012:47(1 PART 1):275-92.

57. HealthPartners. Total cost of care and total resource use validity testing analysis. 2017.

58. Bertoli-Avella AM, Haagsma JA, Van Tiel S, Erasmus V, Polinder S, Van Beeck $E$, et al. Frequent users of the emergency department services in the largest academic hospital in the Netherlands: A 5-year report. Eur J Emerg Med. 2017;24:130-5.

59. Bodenmann P, Baggio S, Iglesias K, Althaus F, Velonaki VS, Stucki S, et al. Characterizing the vulnerability of frequent emergency department users by applying a conceptual framework: a controlled, cross-sectional study. Int J Equity Health. 2015;14:1-10. https://doi.org/10.1186/s12939-015-0277-5.

60. Colligan EM, Pines JM, Colantuoni E, Wolff JL. Factors associated with frequent emergency department use in the medicare population. Med Care Res Rev. 2017;74:311-27.

61. Cunningham A, Mautner D, Ku B, Scott K, LaNoue M. Frequent emergency department visitors are frequent primary care visitors and report unmet primary care needs. J Eval Clin Pract. 2017;23:567-73. 
62. Hardy M, Cho A, Stavig A, Bratcher M, Dillard J, Greenblatt L, et al. Understanding frequent emergency department use among primary care patients. Popul Health Manag. 2017;21. https://doi.org/10.1089/pop. 2017.0030.

63. Kanzaria HK, Niedzwiecki MJ, Montoy JC, Raven MC, Hsia RY. Persistent frequent emergency department use: core group exhibits extreme levels of use for more than a decade. Health Aff. 2017;36:1720-8.

64. Saef SH, Carr CM, Bush JS, Bartman MT, Sendor AB, Zhao W, et al. A comprehensive view of frequent emergency department users based on data from a regional HIE. South Med J. 2016;109:434-9.

65. Lim J. Sustainable health care financing: The Singapore experience. Glob Pol. 2017:8:103-9.

66. Housing \& Development Board. HDB annual report 2017/2018. 2018

67. Hensher DA, Swait JD, Louviere JJ, editors. Choosing a choice model. In: Stated choice methods: analysis and applications. Cambridge: Cambridge University Press; 2000:34-82. doi: https://doi.org/10.1017/ CBO9780511753831.003.

68. Neyman J, Pearson E. On the use and interpretation of certain test criteria for purposes of statistical inference : Part I. Biometrika. 1928;20A:175-240.

69. RStudio Team. RStudio: integrated development for R. 2015. http://www. rstudio.com.

70. Wickham H, Francois R. dplyr: a grammar of data manipulation. 2016. https://cran.r-project.org/package=dplyr.

71. Longman JM, I IM, Passey MD, Heathcote KE, Ewald DP, Dunn T, et al. Frequent hospital admission of older people with chronic disease: a crosssectional survey with telephone follow-up and data linkage. BMC Health Serv Res. 2012;12:1-13.

72. Perkins AJ, Kroenke K, Unützer J, Katon W, Williams JW, Hope C, et al. Common comorbidity scales were similar in their ability to predict health care costs and mortality. J Clin Epidemiol. 2004;57:1040-8.

73. Johnson TL, Rinehart DJ, Durfee J, Brewer D, Batal H, Blum J, et al. For many patients who use large amounts of health care services, the need is intense yet temporary. Health Aff. 2015;34:1312-9. https://doi.org/10.1377/hlthaff. 2014.1186 .

74. Bell J, Turbow S, George M, Ali MK. Factors associated with high-utilization in a safety net setting. BMC Health Serv Res. 2017;17:1-9.

75. Low LL, Yan S, Kwan YH, Tan CS, Thumboo J. Assessing the validity of a data driven segmentation approach : A 4 year longitudinal study of healthcare utilization and mortality. PLoS One. 2018;13:1-15.

76. Davis AC, Shen E, Shah NR, Glenn BA, Ponce N, Telesca D, et al. Segmentation of high-cost adults in an integrated healthcare system based on empirical clustering of acute and chronic conditions. J Gen Intern Med. 2018.

77. Desan PH, Zimbrean PC, Weinstein AJ, Bozzo JE, Sledge WH. Proactive psychiatric consultation services reduce length of stay for admissions to an inpatient medical team. Psychosomatics. 2011;52:513-20. https://doi.org/10. 1016/j.psym.2011.06.002.

78. Wood R, Wand APF. The effectiveness of consultation-liaison psychiatry in the general hospital setting: A systematic review. J Psychosom Res. 2014;76: 175-92. https://doi.org/10.1016/j.jpsychores.2014.01.002.

79. Hussain M, Seitz D. Integrated models of care for medical inpatients with psychiatric disorders: A systematic review. Psychosomatics. 2014;55:315-25. https://doi.org/10.1016/j.psym.2013.08.003.

80. Siddiqui N, Dwyer M, Stankovich J, Peterson G, Greenfield D, Si L, et al. Hospital length of stay variation and comorbidity of mental illness: a retrospective study of five common chronic medical conditions. BMC Health Serv Res. 2018;18:1-10.

81. Hwang W, LaClair M, Camacho F, Paz H. Persistent high utilization in a privately insured population. Am J Manag Care. 2015;21:309-16.

82. Delia D. Mortality, disenrollment, and spending persistence in medicaid and CHIP. Med Care. 2017;55:220-8.

83. Feltner C, Jones CD, Cene CW, Zheng Z, Sueta CA, Coker-Schwimmer EJL, et al. Transitional care interventions to prevent readmissions for persons with heart failure. Ann Intern Med. 2014;160:774-84

84. Phelan EA, Debnam KJ, Anderson LA, Owens SB. A systematic review of intervention studies to prevent hospitalizations of community-dwelling older adults with dementia. Med Care. 2015;53:207-13.

85. Lemmens KMM, Nieboer AP, Huijsman R. A systematic review of integrated use of disease-management interventions in asthma and COPD. Respir Med. 2009;103:670-91. https://doi.org/10.1016/j.rmed.2008.11.017.
86. Kwan J, Sandercock P. In-hospital care pathways for stroke: a cochrane systematic review. Stroke. 2003;34:587-8.

87. Gillespie JJ, Privitera GJ. Bringing patient incentives into the bundled payments model: Making reimbursement more patient-centric financially. Int J Healthc Manag. 2018;0:1-10. https://doi.org/10.1080/20479700.2018.1425276.

88. Busetto L, Luijkx KG, Elissen AMJ, Vrijhoef HJM. Intervention types and outcomes of integrated care for diabetes mellitus type 2: A systematic review. J Eval Clin Pract. 2016;22:299-310.

89. Martínez-González NA, Berchtold P, Ullman K, Busato A, Egger M. Integrated care programmes for adults with chronic conditions: a meta-review. Int J Qual Heal Care. 2014;26:561-70. https://doi.org/10.1093/intahc/mzu071.

90. Damery S, Flanagan S, Combes G. Does integrated care reduce hospital activity for patients with chronic diseases? An umbrella review of systematic reviews. BMJ Open. 2016;6:e011952

91. Baxter S, Johnson M, Chambers D, Sutton A, Goyder E, Booth A. The effects of integrated care: A systematic review of UK and international evidence. BMC Health Serv Res. 2018:18:1-13.

92. World Health Organisation (WHO). Integrated care models: an overview. 2016. http://www.euro.who.int/_data/assets/pdf_file/0005/322475/ Integrated-care-models-overview.pdf.

93. Goodwin N, Smith J, Davies A, Perry C, Rosen R, Dixon A, et al. Integrated care for patients and populations: Improving outcomes by working together. 2012. https://www.kingsfund.org.uk/publications/integrated-carepatients-and-populations-improving-outcomes-working-together.

94. Rinehart DJ, Oronce C, Durfee MJ, Ranby KW, Batal HA, Hanratty R, et al. Identifying subgroups of adult superutilizers in an urban safety-net system using latent class analysis. Med Care. 2018;56:e1-9.

95. Yan S, Kwan YH, Tan CS, Thumboo J, Low LL. A systematic review of the clinical application of data-driven population segmentation analysis. BMC Med Res Methodol. 2018;9:1-12. https://doi.org/10.1186/s12874-018-0584-9.

96. Riley GF. Administrative and claims records as sources of health care cost data. Med Care. 2009;47(Supplement):S51-5.

97. Schousboe JT, Paudel ML, Taylor BC, Kats AM, Virnig BA, Ensrud KE, et al. Estimating true resource costs of outpatient care for medicare beneficiaries: Standardized costs versus medicare payments and charges. Health Serv Res. 2016;51:205-19.

98. Taira DA, Seto TB, Siegrist R, Cosgrove R, Berezin R, Cohen DJ. Comparison of analytic approaches for the economic evaluation of new technologies alongside multicenter clinical trials. Am Heart J. 2003;145:452-8.

99. Saxena N, You AX, Zhu Z, Sun Y, George PP, Teow KL, et al. Singapore's regional health systems-a data-driven perspective on frequent admitters and cross utilization of healthcare services in three systems. Int J Health Plann Manage. 2017;32:36-49.

\section{Publisher's Note}

Springer Nature remains neutral with regard to jurisdictional claims in published maps and institutional affiliations.

Ready to submit your research? Choose BMC and benefit from:

- fast, convenient online submission

- thorough peer review by experienced researchers in your field

- rapid publication on acceptance

- support for research data, including large and complex data types

- gold Open Access which fosters wider collaboration and increased citations

- maximum visibility for your research: over $100 \mathrm{M}$ website views per year

At $\mathrm{BMC}$, research is always in progress.

Learn more biomedcentral.com/submission 